\title{
Deleterious Effects of Alcohol Intoxication: Diminished Cognitive Control and Its Behavioral Consequences
}

\author{
Todd S. Casbon \\ Florida State University \\ Alan R. Lang \\ Florida State University
}

\author{
John J. Curtin \\ University of Wisconsin-Madison \\ Christopher J. Patrick \\ University of Minnesota
}

\begin{abstract}
The authors tested the hypothesis that impaired behavioral performance during intoxication results partly from alcohol's deleterious effects on cognitive control. The impact of alcohol on perseverative behavior was examined with an $n$-back working memory task that included manipulations of task complexity and prepotency of inclinations to respond or withhold responding. Thirty-two social drinkers (16 men) participated in either an alcohol $(.075 \mathrm{~g} / 100 \mathrm{ml})$ or a no-alcohol condition. Alcohol increased perseveration of prepotent, task-inappropriate response patterns only under cognitively demanding (heavy memory load) conditions. This effect was evident for both commission errors (response persistence despite contingencies altered to require restraint) and omission errors (failure to respond when contingencies were revised to encourage action). Findings suggested that alcohol-induced perseveration arises from impairments in cognitive control.
\end{abstract}

Although the general deleterious effects of alcohol on behavioral performance have been recognized for decades (e.g., Conger, 1951; Jellinek \& McFarland, 1940; Mitchell, 1985), the specific mechanisms by which alcohol exerts these effects have not been established. Progress on this front has probably been hampered by some of the same difficulties often encountered in efforts to establish specific versus general deficits associated with other conditions or disorders (cf. Chapman \& Chapman, 1973, 2001; Knight \& Silverstein, 2001; Strauss, 2001). Nonetheless, some recent work has made significant contributions to the specification of the contextual conditions under which alcohol-induced impairments in behavioral control are most likely to arise (e.g., Curtin, Patrick, Lang, Cacioppo, \& Birbaumer, 2001; Fillmore, VogelSprott, \& Gavrilescu, 1999; Holloway, 1994; Melia, Corodimas, Ryabinin, Wilson, \& LeDoux, 1994) and has begun to provide

Editor's Note. Kenneth Sher served as the action editor for this article.TBB

Todd S. Casbon and Alan R. Lang, Department of Psychology, Florida State University; John J. Curtin, Department of Psychology, University of Wisconsin-Madison; Christopher J. Patrick, Department of Psychology, University of Minnesota.

The work was supported by Grant AA12164 from the National Institute on Alcohol Abuse and Alcoholism, and Grants MH48657, MH52384, and MH65137 from the National Institute of Mental Health. This article was based on research conducted by Todd S. Casbon, under the supervision of Alan R. Lang, in partial fulfillment of the requirements for a master of science degree.

Correspondence concerning this article should be addressed to Alan R. Lang, Department of Psychology, Florida State University, Tallahassee, Florida 32306-1051. E-mail: allang@psy.fsu.edu clues suggesting the particular cognitive deficits apt to be associated with acute alcohol intoxication.

These studies have shown that when stimuli are simple or explicit, are presented in the absence of competing demands, and involve automatic processes linked to immediate responses, effects of alcohol on behavior appear to be limited. Simple psychomotor performance or reactions to salient sensory stimuli or explicitly conditioned cues exemplify such conditions. In contrast, behavioral effects of drinking are often observed in situations involving abstract or complex contextual stimuli, competition for processing resources, delayed responding, and shifting response contingencies. Hence, a common feature of tasks in which alcohol effects are reliably observed is that they seem to require cognitive control, suggesting that alcohol may have specific effects on this function and the brain processes that underlie it.

The idea that cognitive impairments underlie the "disinhibitory" effects of alcohol has also been advanced in accounts of how drinking can affect socially significant (e.g., sexual and aggressive) behaviors that entail competing approach and avoidance contingencies (e.g., Steele \& Josephs, 1990). Once momentum toward a particular action tendency-for instance, approach with an expectation of immediate gratification-is instigated in an intoxicated individual, it has a greater likelihood of persisting despite cues that would normally prompt inhibition. We use the term perseveration to refer to such continuance of a behavior pattern when other signals call for its alteration or cessation. Although perseveration is generally associated with impulsive errors of commission (i.e., persistence of active responding when current conditions call for restraint), impaired cognitive control could also result in errors of omission (i.e., failure to enact responses that have become appropriate due to a shift in contingencies). Commission errors may underlie behavioral excesses linked with drinking, whereas victimization or other loss stemming from 
failure to take adaptive action may reflect errors of omission. Although they may be mediated by different underlying brain structures, both types of outcome are predictable consequences of diminished cognitive control.

This report describes the results of an experiment designed to elucidate alcohol's effects on behavioral performance by examining the role of two distinct aspects of cognitive control in perseverative responding: working memory and set-shifting processes. To achieve this, we assessed increases in perseverative behavior during intoxication as a function of two task manipulations: cognitive load and response prepotency. We begin with a specific description of set-shifting/response prepotency because they have been less frequently elaborated in the research literature than working memory/cognitive load, and thus are likely to be less familiar to readers. Efforts have been made to integrate descriptions of working memory/cognitive load throughout the text.

\section{Response Prepotency and Perseveration}

A prepotent response tendency can be defined as a strong, essentially automatic inclination to select or implement one response option over others. Prepotency can arise for a number of reasons, including training or practice, emotional or physiological states favoring a particular response option, and incentives or contingencies associated with a certain response. The inclination to read words in a standard Stroop task is one example. Participants viewing color words displayed in varying colors of ink show a delay in naming the ink color on trials involving a mismatch between the word itself and the color in which it appears, presumably because the inclination to read words is stronger or more automatic than the inclination to name colors. Compared to color naming, word reading is an everyday activity that is extensively rehearsed and reinforced over time by the immediate rewards associated with being able to read. Thus, the Stroop task involves competition between an automatic, but task-inappropriate inclination to read the word, and a more controlled effort to name the color and thereby avoid errors.

From this perspective, perseverative behavior can be viewed as the persistence of a previously task-appropriate response option, automatized through repetition, despite revised contingencies that mandate a different response. The likelihood of perseveration should increase as a function of the extent of rehearsal of the previously appropriate, but currently inappropriate, response.

\section{The Role of Cognitive Control in Regulating Response Prepotency and Perseveration}

Although researchers have described the cognitive control construct in various ways (e.g., Baddeley \& Della Salla, 1996; Botvinick, Braver, Barch, Carter, \& Cohen, 2001; Braver, Barch, \& Cohen, 1999; Goldman-Rakic, 1996; Norman \& Shallice, 1986), we focus here on the conceptualization outlined by Cohen and colleagues because it directly addresses the role of cognitive control in overriding prepotent response inclinations. Cohen and colleagues defined cognitive control as the ability to guide behavior flexibly by internally represented goals or intentions that support task-relevant processes and responses and allow them to compete against irrelevant responses (Braver et al., 1999; MacDonald, Cohen, Stenger, \& Carter, 2000). A high degree of cog- nitive control is required to (a) address novel, complex, or otherwise difficult tasks, (b) overcome habitual or prepotent responses, and (c) correct errors (MacDonald et al., 2000).

Within this conceptualization, effective task-appropriate responding depends on at least two interrelated, but conceptually distinct processes: working memory and set shifting. Cohen, Barch, Carter, and Servan-Schreiber (1999) stressed the importance of the former in maintaining and applying context representations to guide task-appropriate behavior. Context representations can include information about specific stimuli encountered previously, representations corresponding to the product of processing of a prior stimulus sequence, or more abstract information such as task instructions. Thus, task-appropriate responding depends upon the extent to which the individual can use working memory to store and update global task instructions regarding stimulusresponse mappings, as well as representations of specific contextual stimuli. As task complexity increases (e.g., stimulus-response relations become more intricate; delays between stimulus and response are longer), a greater load is placed on working memory resources, which decreases sensitivity to differences between stimuli linked to separate response options. When sensitivity is diminished, stimulus discrimination suffers, and the likelihood of taskinappropriate behavior increases.

The second control process required for task-appropriate behavior is set shifting, that is, the ability to direct behavior away from one response option toward another more appropriate option based on current task contingencies. To achieve this, the respondent must suppress activation associated with a previously appropriate response and selectively activate the alternative, currently appropriate response. If one response has become prepotent through repetition, the strength of its activation may bias that response to persist even when the stimulus context calls for a different response. The ability to overcome a prepotent response tendency depends upon adequate activation of global task instructions that prepare the respondent for a behavioral shift when the current stimulus context dictates an alternative response according to those instructions. Even when working memory is intact, ensuring sensitivity to differences among task stimuli, the presence of a prepotent but task-inappropriate response may bias the respondent toward errors. However, impairments in working memory would be expected to exacerbate perseverative errors, given that global representations of task instructions, which prepare the individual for behavioral shifts, must be held in working memory alongside representations of the current stimulus context.

The foregoing account suggests that reliance on control processes for guidance of appropriate behavior should be most important in tasks that (a) are complex, (b) involve competition between prepotent and alternative response inclinations, and (c) require effective representations of contextual cues or information. These are precisely the conditions under which alcohol has been shown to have its most profound behavioral effects, thereby suggesting that alcohol-induced impairment of cognitive control processes may account for its disinhibitory effects on behavior.

This hypothesis was supported by a recent study showing that alcohol decreased the influence of controlled processes on intentional behavior in a word-stem completion task, while leaving intact the influence of automatic processes (Fillmore et al., 1999). Those investigators used a procedure devised by Jacoby, Jennings, and Hay (1996) and Jacoby, Toth, and Yonelinas (1993) to disso- 
ciate the influence of controlled and automatic processes on intentional behavior. The underlying premise was that when controlled and automatic processes work in concert to achieve the same goal, task performance is facilitated, but when they work in opposition, competing response inclinations (i.e., prepotent or automatic vs. weaker inclinations that require control) emerge. Under the latter condition, impairment of intentional behavior is likely if the influence of controlled processes is not sufficiently strong to guide the weaker task-relevant response effectively.

The work of Fillmore et al. (1999) represents a process-oriented approach to the study of cognitive mechanisms underlying alcohol-induced behavioral impairment. In the process-oriented approach, evidence for specific cognitive deficits is provided by demonstrating specific, theoretically predictable patterns of performance across different experimental conditions (see Knight \& Silverstein, 2001, for a detailed account of this approach). In this study, we also used a process-oriented approach to further investigate the role of specific cognitive deficits in the perserverative behavior of alcohol-intoxicated individuals.

\section{Study Design and Predictions}

\section{Tasks}

We assessed the performance of intoxicated and sober participants in a task designed to manipulate two specific variables relevant to cognitive control as conceptualized by Cohen and colleagues (1999): the cognitive complexity of the task and the prepotency of tendencies to respond and to withhold responding. Indices of sensitivity and response bias, derived using signal detection theory (SDT), were used to index working memory and set-shifting functions, respectively, and specific hypotheses were tested regarding alcohol's impact on these indices and on performance across varying task conditions.

The task was a variant of the " $n$-back" working memory paradigm, a well-validated procedure for manipulating working memory load. The $n$-back task involves concurrent storage and manipulation of information, processes emphasized in contemporary theories of working memory (e.g., Baddeley, 1986). Our choice of this task was based on consensus among cognition researchers that working memory plays an essential role in complex cognition (e.g., Baddeley, 1986; Goldman-Rakic, 1992; Haberlandt, 1997; Jonides, 1995; Shah \& Miyake, 1999), and the fact that memory load can be effectively manipulated using this task.

In the $n$-back task, participants receive specific instructions regarding when to respond with a button press to a target alphabetic character and when to withhold responses. They then view a series of target and nontarget characters presented in succession on a computer screen. Variations in the instructions permitted us to assess alcohol's effects under differing levels of memory load (light, heavy), manipulated across trial blocks within subjects. In the light load (1-back) condition, participants were instructed to determine whether the current stimulus matched the immediately preceding stimulus, and then respond according to instructions; in the heavy load (2-back) condition, participants were instructed to determine whether the current stimulus matched the stimulus two positions back, and then respond according to instructions.

Contingencies for the task were also arranged so that an active response was required to $80 \%$ of stimuli in designated trial blocks at each memory load, and only $20 \%$ of stimuli in other blocks. This within-subjects response frequency manipulation permitted assessment of the biasing effect of a prepotent behavioral set to either respond ( $80 \%$ blocks) or withhold responding ( $20 \%$ blocks) across trials within blocks.

Consistent with a process-oriented approach, we predicted that alcohol would produce a specific pattern of effects on task accuracy (i.e., perseverative behavior) and signal detection parameters of sensitivity and response bias as a function of memory load and behavioral prepotency. Hypotheses for perseverative errors and signal detection parameters follow.

\section{Predictions}

1. Perseveration. It was predicted that the behavioral set established in an ongoing trial block via manipulation of expected response frequency within the block would lead to increased errors in intoxicated participants under conditions of heavy memory load. Specifically, a Beverage $\times$ Memory Load $\times$ Block Response Frequency interaction was predicted for both commission and omission errors. Decomposition of this interaction was expected to reveal: (a) increased commission error rates ${ }^{1}$ for intoxicated individuals under heavy memory load in $80 \%$ response frequency blocks (where the prepotent set is to respond) and (b) increased omission error rates $^{2}$ for intoxicated participants under heavy memory load in $20 \%$ response frequency blocks (where the prepotent set is to withhold response).

In connection with these hypothesized effects for alcohol-induced perseverative behavior, we predicted specific patterns of working memory impairment (i.e., SDT sensitivity) and set shifting (i.e., SDT response bias) processes within these same task conditions. Specifically, hypotheses for the SDT parameters were as follows:

2. Sensitivity. The SDT sensitivity parameter indexes the ability to discriminate between target and nontarget events. We predicted that sensitivity would be reduced in the 2-back versus the 1-back condition because the increased memory demands associated with the former should add difficulty to discrimination between match and nonmatch trials. Furthermore, based on evidence that alcohol impairs working memory capacity (Finn, Justus, Mazas, \& Steinmetz, 1999), we predicted a significant Beverage Group $\times$ Memory Load interaction for sensitivity. Decomposition of this interaction was expected to reveal reduced sensitivity for alcohol participants specifically in the heavy load condition. The behavioral prepotency manipulation was not expected to affect sensitivity.

3. Response bias. The SDT response bias parameter indexes preparedness to respond in one direction or another. Accordingly, we predicted a main effect of response prepotency, but not memory load, on this SDT parameter. However, we did expect the impact of the prepotency manipulation to be greater under conditions of stimulus uncertainty associated with higher memory load, and therefore a

\footnotetext{
${ }^{1}$ Commission error rates were calculated as the total number of trials on which a button press was made when it should not have been made, divided by the total number of trials on which the correct action was to withhold a response.

${ }^{2}$ Omission error rates were calculated as the total number of trials on which a button press was not made when it should have been made, divided by the total number of trials on which the correct action was to respond with a button press.
} 
significant Memory Load $\times$ Block Response Frequency interaction was also predicted. Furthermore, we hypothesized that alcohol would enhance the biasing effect of the block frequency manipulation, particularly in the high memory load condition. Thus, a Beverage Group $\times$ Memory Load $\times$ Block Response Frequency interaction was predicted for response bias. Decomposition of this interaction was expected to reveal: (a) increased bias to respond for intoxicated individuals under heavy memory load in $80 \%$ response frequency blocks (where the prepotent set is to respond) and (b) increased bias to withhold response for intoxicated participants under heavy memory load in $20 \%$ response frequency blocks (where the prepotent set is to withhold response).

\section{Method}

\section{Participants}

Thirty-two undergraduate students (16 men) were recruited from introductory psychology classes at Florida State University. Participants were required to be at least 21 years old, have recent experience with doses of alcohol comparable to those to be administered in our study, have no history of alcohol-related problems or any medical condition that might contraindicate alcohol consumption, and have access to safe transportation home after the experiment. Only 3 prospective participants were excluded by these criteria: 1 due to a regimen of a contraindicated prescription medication, 1 due to a self-reported history of alcohol problems on pertinent screening instruments, and 1 due to alcohol consumption that exceeded maximum inclusion criteria (viz., $>5$ drinks/day for men or $>4$ drinks/day for women). All remaining eligible volunteers were scheduled and instructed to abstain from any alcohol for at least $24 \mathrm{hr}$, from any other drugs for at least $72 \mathrm{hr}$, and from all food and beverages for at least $4 \mathrm{hr}$ prior to arrival for testing. Compensation for participation involved receipt of class research participation credit up to $3 \mathrm{hr}$, plus payment at the rate of $\$ 5 / \mathrm{hr}$ for any time past $3 \mathrm{hr}$. All procedures for the study were fully approved by the Florida State University Institutional Review Board.

\section{Self-Report Individual Difference Measures of Alcohol Use}

Drinking History Questionnaire (DH). Participants completed a modified version of this instrument (Cahalan, Cisin, \& Crossley, 1969), containing 10 items that assess the quantity and frequency of current and past alcohol consumption as well as subjective experiences and beliefs regarding the individual's own use of alcohol. Because individuals with heavier drinking histories may have developed greater tolerance to alcohol's impairing effects than individuals who tend to drink less, this measure was included to verify comparable drinking histories between beverage groups.

Short Michigan Alcoholism Screening Test-Self, Father, and Mother (S-SMAST, Selzer, Vinokur, \& Van Rooijen, 1975; F-SMAST and M-SMAST, Crews \& Sher, 1992). These 13-item measures provide a reliable index of self, paternal, and maternal drinking problems, respectively, with scores of 3 or higher indicative of a personal or parental problem drinking. The S-SMAST was included to facilitate identification and exclusion of problem drinkers from study participation, although no actual study participants received a score of 3 or higher (likely because such individuals had already been excluded in earlier stages of screening). The F-SMAST and M-SMAST measures were included because there has been some evidence to suggest differential sensitivity to alcohol's effects based on family history of alcoholism (Holloway, 1994), although this evidence is somewhat equivocal.

\section{Procedure}

Consent and screening. Upon arrival at the laboratory, all participants were seated in a comfortable room where drinking would later occur. All were required to provide proof of legal drinking age (valid driver's license) and sign consent forms that included an agreement to remain at the research site until their blood alcohol levels (BALs) were sufficiently low to permit safe release. They also completed a drinking and medical history questionnaire. All women completed a urine sample pregnancy test (QuickVue One-Step hCG; Quidel, San Diego, CA) during this initial period, with a negative result required for further participation. Qualified participants were then asked to provide a breath sample to verify an initial BAL of 0.00 (BAC Verifier; Verax Corporation, Fairport, NY).

Beverage manipulation. Half the male and half the female participants were randomly assigned to the alcohol group, the remainder to the noalcohol group. The alcohol group received a beverage consisting of fruit juice mixed with 95\% ethyl alcohol in a 7:1 juice-to-alcohol ratio. They were accurately informed of their beverage condition and were told that the dose was roughly equivalent to three or four standard drinks in $1 \mathrm{hr}$ for a 150-lb. (approximately $68-\mathrm{kg}$ ) person. The dose required to produce a maximum target peak BAL $(0.075 \mathrm{~g} / 100 \mathrm{ml}) 30 \mathrm{~min}$ after the completion of beverage consumption was computed for each participant using a computer program (Curtin \& Verona, 1996) developed for this purpose. ${ }^{3}$ Participants assigned to the no-alcohol group were correctly advised of it, ${ }^{4}$

\footnotetext{
${ }^{3}$ The procedure used to determine alcohol dosage in this study was developed using formulas available from Watson (1989). It is predicated on the assumption that to reach a target BAL, the alcohol dose to be administered is a function of the participant's height, weight, age, gender, total body water (TBW), duration of the drinking period (DDP), time to peak BAL (TPB), and alcohol metabolism rate (MR). More specifically,
}

Alcohol dose $(\mathrm{g})=(10 \cdot \mathrm{BAL} \cdot \mathrm{TBW}) / 0.8$

$$
+10 \cdot \mathrm{MR} \cdot(\mathrm{DDP}+\mathrm{TPB}) \cdot(\mathrm{TBW} / 0.8) \text {. }
$$

We used $0.015 \mathrm{~g} / 100 \mathrm{ml} / \mathrm{hr}$ as the average metabolism rate for all participants. In addition, we assumed that participants reached their peak BAL at $0.5 \mathrm{hr}$ after cessation of drinking. TBW was determined separately for men and women using gender-specific regression equations provided by Watson:

$$
\begin{gathered}
\text { Men's TBW }=2.447-0.09516 \cdot \text { age }+0.1074 \\
\cdot \text { height }(\text { in } \mathrm{cm})+0.3362 \cdot \text { weight }(\text { in } \mathrm{kg}) \\
\text { Women's TBW }=-2.097+0.1069 \\
\cdot \text { height (in } \mathrm{cm})+0.2466 \cdot \text { weight }(\text { in } \mathrm{kg}) .
\end{gathered}
$$

${ }^{4}$ The decision to use a no-alcohol, as opposed to a placebo, comparison group was a reasoned one. First, because this study was the initial effort to examine experimentally the role of cognitive complexity and prepotent response inclinations in alcohol-induced perseveration of behavior, we believed that the use of an ecologically valid, extreme-groups design was justified. It seemed to make sense to demonstrate that there was an effect unique to the combination of accurately anticipated alcoholic beverage consumption, heavy working memory load, and prepotent response inclinations before turning to a more fine-grained analysis of all of the possible causal mechanisms, including expectancy versus pharmacology, that might underlie it. Second, our ability to rule out global expectancy effects was strengthened by our prediction of differential alcohol effects across light versus heavy memory load conditions, $80 \%$ versus $20 \%$ block response frequency conditions, and $20 \%$ first versus $20 \%$ after $80 \%$, and $80 \%$ first versus $80 \%$ after $20 \%$, response frequency order conditions. Although it was reasonable for participants to hold the expectation that alcohol could impair overall performance on the $n$-back task, it seemed improbable that they would expect differential impairment across variations of each of these separate independent variables. Finally, we were concerned about the possible impact of participants' suspicions about placebo manipulations on the critical cognitive load and cognitive capacity constructs. 
and received only a mixture of fruit juice in a volume equivalent to the total amount that would have been administered had they been in the alcohol condition. All beverages were evenly divided into two drinks, each consumed in $10 \mathrm{~min}$, for a total drinking period of $20 \mathrm{~min}$; a 10-min absorption period followed the drinking. Participants then completed their first postbeverage breath test prior to beginning the $n$-back working memory task.

The $n$-back working memory task. The $n$-back task consists of a series of 32 one-minute trial blocks, each involving the presentation of a series of 20 letters. Following the method used by Jonides, Schumacher, Smith, \& Lauber (1997), the letters were pseudorandom sequences of consonants other than L, W, and Y, randomly varying in case. Each letter stimulus was presented for $500 \mathrm{~ms}$, with a 2,500-ms intertrial interval (ITI). The letters measured $1 \mathrm{in.}(2.54 \mathrm{~cm})$ in height and were presented in the center of a computer monitor in white print against a black background. The presentation and timing of the letter stimuli and the collection of behavioral response data were controlled by a PC computer running the Virtual Psychophysiology Monitor (VPM) software package (Cook, Atkinson, \& Lang, 1987). Behavioral responses were made with a handheld response button connected to the computer.

The task included light and heavy memory load conditions. In the light load condition, participants were instructed to decide whether the current letter stimulus, regardless of case, matched the letter immediately preceding it (i.e., one trial back). The heavy load condition required participants to determine whether the current stimulus matched the one presented two trials back. Letter case (upper or lower) was varied randomly to ensure that participants determined matches based on the identities or names of the letters and not their physical forms. Half of the 32 blocks were light load (1-back) blocks and half were heavy load (2-back) blocks. Across all trial blocks, $20 \%$ of stimuli matched according to memory load instructions and $80 \%$ did not match.

To manipulate block response frequency within subjects, participants were instructed in some blocks to respond only when the current stimulus matched the one $n$ trials back (i.e., 20\% response frequency), and in others to respond to every stimulus unless it matched the one presented $n$ trials back (i.e., $80 \%$ response frequency). Within each of the two memory load conditions, half of the 16 blocks had a response frequency of $20 \%$ and half had a response frequency of $80 \%$. Reflecting the differing memory load and response frequency conditions, the 32 task blocks were divided into eight blocks each of four types: (a) light load/20\% response frequency, (b) light load $/ 80 \%$ response frequency, (c) heavy load/20\% response frequency, and (d) heavy load/80\% response frequency. For counterbalancing purposes, the order of occurrence of blocks of each type was varied such that the two light $(\mathrm{L})$ load types either preceded or followed the two heavy $(\mathrm{H})$ load types (i.e., either L-L-H-H, or H-H-L-L). Within each of the two memory conditions, all $20 \%$ response blocks occurred either first (before all $80 \%$ response blocks) or second (after all $80 \%$ response blocks). Combining the two memory load conditions and the two response frequency conditions in this manner yielded four different task orders, across which the sequencing of blocks of each type was balanced (see Table 1). Within each beverage group, an equal number of participants was assigned to each task order.

Table 1

Four Different Stimulus Orders Used for Counterbalancing Purposes

\begin{tabular}{cll|ll}
\hline Order & Blocks 1-8 & Blocks 9-16 & Blocks 17-24 & Blocks 25-32 \\
\hline 1 & Light $/ 20 \%$ & Light/80\% & Heavy/20\% & Heavy/80\% \\
2 & Light/80\% & Light/20\% & Heavy/80\% & Heavy/20\% \\
3 & Heavy/20\% & Heavy/80\% & Light $/ 20 \%$ & Light $/ 80 \%$ \\
4 & Heavy/80\% & Heavy/20\% & Light/80\% & Light $/ 20 \%$ \\
\hline
\end{tabular}

Note. The vertical rule denotes the split between memory load levels for each of the four orders.
Experimental task instructions. Before beginning the task, participants were given instructions appropriate to their initial block type, and were advised to respond both as quickly and as accurately as possible to task stimuli. Next, a brief practice exercise was administered to make clear the specific task requirements for that block type and to ensure understanding of the instructions. The experimenter displayed a card on which eight consecutive letters (i.e., g, B, b, b, C, b, B, C) were listed and, pointing sequentially to each letter, asked the participant to state whether or not a response was called for by the current instructions. The actual computerized task did not begin until the participant was able to complete the practice exercise perfectly. Most participants, including those who had consumed alcohol, required no more than two or three attempts to meet this criterion and many were able to do so on the first attempt.

After completing the practice exercise for blocks of a designated type, participants completed eight consecutive blocks of that type, consisting of 20 letter trials each. At the end of each block, the participant was given veridical feedback regarding response accuracy. After completing eight consecutive blocks of a given type, a breath alcohol test was administered, and the participant was then given instructions and a practice for the next block type. This procedure was repeated until the participant completed all eight blocks of each of the four types.

Debriefing and dismissal. Following completion of the $n$-back working memory task, participants were debriefed, and those in the no-alcohol condition were dismissed. Those who had received alcohol completed additional BAL tests until two consecutive readings were below established criteria for release, at which time they were driven or escorted home.

\section{Results}

\section{Beverage Administration Manipulation Check}

All participants registered a BAL of zero upon arrival at the laboratory. Participants in the alcohol beverage condition achieved BALs of .051, .062, .066, .066, and $.063 \mathrm{~g}$ per $100 \mathrm{ml}$, respectively, across the five subsequent assessment points (i.e., immediately prior to the start of the main experimental task, and after completion of $8,16,24$, and 32 experimental trial blocks). A Gender $\times$ Time $(5$ postbeverage BAL assessment points) repeated-measures analysis of variance (ANOVA) revealed a main effect of Time, $F(4,11)=12.41, p<.001$. Neither the main effect of gender nor the Gender $\times$ Time interaction was significant, indicating that both genders achieved comparable BALs with a similar time course across the experiment. ${ }^{5}$ Decomposition of the main effect of time into simple, Bonferroni-corrected, pairwise comparisons indicated that the BAL immediately prior to the start of the task was significantly lower than all subsequent BALs (all $t \mathrm{~s} \geq 3.76$ ). No significant differences between BALs were observed among any other assessment point comparisons. Thus, BAL was either rising (initially) or flat throughout testing.

\section{Individual Differences in Alcohol Use}

Participants in the alcohol and no-alcohol groups were compared on demographic (age, race, gender), drinking history (frequency and quantity of drinking, drinks per week), and self/ parental problems with drinking (SMAST scores). Descriptive statistics for the two groups are presented in Table 2. No signifi-

\footnotetext{
${ }^{5}$ No significant main effects or interactions with gender were observed in any of the reported analyses. Therefore, all subsequent results are reported collapsed across gender.
} 
cant differences were observed between the two beverage groups on any of these individual-difference variables.

\section{Data-Analytic Strategy}

Analysis of each primary dependent variable (i.e., error rates and signal detection indices) was accomplished within separate repeated-measures analyses with beverage (alcohol vs. no-alcohol) as a between-subjects variable and memory load (light vs. heavy) and block response frequency (20\% response vs. $80 \%$ response) as within-subjects variables. When simple effect tests were required to decompose complex interactions, Bonferroni-corrected alphas were utilized to control for inflation of family-wise Type I error. In these instances, the corrected alpha level is provided in parentheses following the description of the simple effect test.

\section{Error Rate Analyses}

Beverage effects on error rates (commission and omission errors) during $n$-back task performance) were examined within a doubly multivariate repeated-measures Beverage $\times$ Memory Load $\times$ Block Response Frequency multivariate analysis of variance (i.e., a repeated-measures MANOVA with, in this case, two dependent variables; see SPSS Advanced Models 10.0, 1999). Commission and omission error rates were included simultaneously as dependent variables in this MANOVA to provide further protection against inflation of Type I error when analyzing related dependent variables for which comparable independent variable effects are predicted (Stevens, 2002). Figure 1 depicts rates of commission (top panel) and omission errors (bottom panel) as a function of beverage, memory load condition, and block response frequency.

As expected, significant multivariate main effects of beverage, $F(2,29)=5.90, p=.007$, memory load, $F(2,29)=37.03, p<$ .001 , and block response frequency, $F(2,29)=41.42, p<.001$ were observed. However, all main effects (and lower order inter-

Table 2

Individual Difference Measures by Beverage Condition

\begin{tabular}{lcc}
\hline \multicolumn{1}{c}{ Measure } & No-alcohol & Alcohol \\
\hline $\begin{array}{l}\text { Demographic information } \\
\text { Age (years) }\end{array}$ & $23.0(2.3)$ & $22.8(2.3)$ \\
Gender & & \\
$\quad$ Female & $50 \%(n=8)$ & $50 \%(n=8)$ \\
$\quad$ Male & $50 \%(n=8)$ & $50 \%(n=8)$ \\
Race & & \\
$\quad$ Caucasian & $87 \%(n=14)$ & $81 \%(n=13)$ \\
$\quad$ Other & $13 \%(n=2)$ & $19 \%(n=3)$ \\
Drinking history & $2.0(1.3)$ & $2.4(1.7)$ \\
$\quad$ Frequency (occasions/week) & $4.4(2.0)$ & $4.1(1.7)$ \\
$\quad$ Quantity (drinks/occasion) & $8.7(7.2)$ & $8.8(4.7)$ \\
$\quad$ Drinks/week & $0.4(0.8)$ & $0.7(0.8)$ \\
SMAST scores & $0.4(1.3)$ & $1.2(2.4)$ \\
$\quad$ Self & $0.3(1.0)$ & $1.7(2.8)$ \\
$\quad$ Father & & \\
$\quad$ Mother &
\end{tabular}

Note. Data are means (and standard deviations) unless otherwise indicated. SMAST $=$ Short Michigan Alcoholism Screening Test. No contrasts between alcohol and no-alcohol groups were statistically significant at $\alpha=$ .05 .
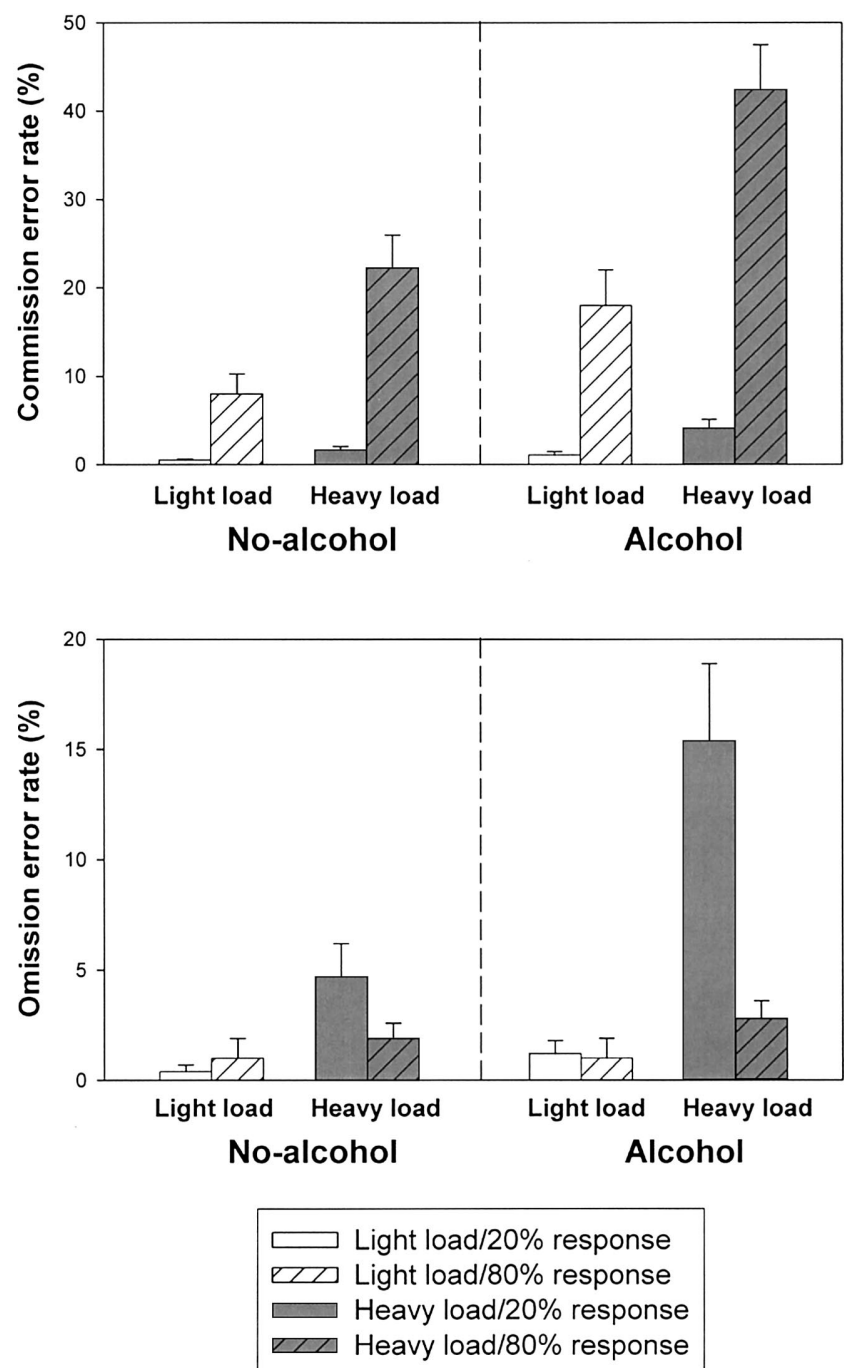

Figure 1. Mean commission error rates (top panel) and omission error rates (bottom panel) by beverage group, memory load (light load $=1$-back, heavy load $=2$-back), and block response frequency.

actions ${ }^{6}$ ) were moderated by the predicted Beverage $\times$ Memory Load $\times$ Block Response Frequency interaction, $F(2,29)=4.61$, $p=.018$. To understand how beverage effects were moderated by memory load and block response frequency in this three-way interaction, simple effects tests of beverage were conducted separately for commission and omission error rates across the four combinations of memory load and block response frequency (i.e., light load $/ 20 \%$, light load/ $80 \%$, heavy load $/ 20 \%$, and heavy load/ $80 \% ; \alpha=.05 / 4=.0125)$.

For commission errors, a significant beverage effect was found only during heavy load $/ 80 \%$ response trials, $t(30)=3.26, p=$

\footnotetext{
${ }^{6}$ All two-way interactions were significant. However, these lower-order interactions are subsumed by the significant three-way Beverage $\times$ Memory Load $\times$ Block Response Frequency interaction. Therefore, interpretation of effects is most parsimoniously accomplished by decomposition of this three-way interaction.
} 
.003 , reflecting greater errors of this type among intoxicated than nonintoxicated participants specifically under heavy load, frequent response contingencies $(M \mathrm{~s}=42.4 \%$ and $22.2 \%$, respectively; see Figure 1, top panel). For omission errors, the simple effect of beverage was significant only during heavy load $/ 20 \%$ response trials, $t(30)=2.85, p=.008$, reflecting greater errors of this type among alcohol than no-alcohol participants exclusively under heavy load, infrequent response contingencies $(M \mathrm{~s}=15.4 \%$ and $4.7 \%$, respectively; see Figure 1, bottom panel). There was no significant effect of beverage on errors of either type for any other combination of memory load and response frequency.

These selective effects of alcohol on commission and omission error rates highlight two key points. First, alcohol significantly enhanced the frequency of errors only when high demands were placed on working memory resources (i.e., during heavy load, 2-back trials). Second, the specific behavioral impairment (i.e., type of error) during heavy memory load was dependent upon the currently prepotent behavioral set. In $80 \%$ response frequency blocks, which encouraged an active response set, intoxicated participants were inclined to continue responding even when inappropriate (i.e., commission errors, see Predictions). Conversely, in $20 \%$ response frequency blocks, which encouraged the withholding of response, intoxicated participants failed to respond when appropriate (i.e., omission errors, see Predictions). In both instances, intoxication under heavy working memory load resulted in a perseveration of the prevailing prepotent response set derived from the current response frequency context.

\section{Signal Detection Analyses}

Signal detection theory (SDT; Green \& Swets, 1966; Macmillan \& Creelman, 1991) holds that two conceptually distinct response determinants, sensitivity and response bias, contribute to the errors observed during the $n$-back task in this experiment. Sensitivity reflects the ability to discriminate accurately between conditions (match vs. mismatch trials). In the $n$-back task, this is primarily an index of working memory function. In contrast, response bias reflects a predisposition to select differentially one response option (i.e., respond vs. withhold response) over the other, independent of discriminative ability. Numerous specific SDT indices have been proposed, but for our purposes, we selected $d^{\prime}$ and $c^{\prime}$ to measure sensitivity and response bias, respectively. ${ }^{7}$ Examination of beverage effects on $d^{\prime}$ and $c^{\prime}$ indices permitted disaggregation of the processes contributing to errors so that the specific and independent effects of experimental manipulations on working memory function and response prepotency could be examined.

Sensitivity. Means and standard errors across all conditions for the SDT $d^{\prime}$ index of sensitivity ${ }^{8}$ are displayed in the top panel of Figure 2. As expected, a significant main effect of memory load was observed, with reduced sensitivity (i.e., impaired ability to discriminate between match and mismatch trials) observed during heavy (2-back) vs. light (1-back) memory load conditions, $F(1$, $30)=92.09, p<.001$. A significant main effect of block response frequency was also obtained, with reduced sensitivity during the $80 \%$ response blocks relative to the $20 \%$ response blocks, $F(1$, $30)=31.72, p<.001$. A Memory Load $\times$ Block Response Frequency interaction was also observed, $F(1,30)=5.07, p=$ .032 , with the combination of heavy memory load and $80 \%$ response frequency resulting in a somewhat greater than additive reduction in sensitivity.
The main effect of memory load was also moderated by a Beverage $\times$ Memory Load interaction, $F(1,30)=7.17, p=.012$. Bonferroni-corrected simple effects tests of memory load within each beverage $(\alpha=.05 / 2=.025)$ were conducted to examine this effect. Significant simple effects of memory load, with reduced sensitivity in heavy, relative to light, load condition, were observed within both the alcohol, $t(15)=10.20, p<.001$, and no-alcohol, $t(15)=4.33, p<.001$, groups. Importantly, however, the interaction indicated that the memory load effect on sensitivity was larger in the alcohol group.

A significant main effect of beverage, $F(1,30)=8.76, p<.006$ was also moderated by the Beverage $\times$ Memory Load interaction, $F(1,30)=7.17, p=.012$. Therefore, beverage effects were examined with simple effects tests of beverage at each memory load $(\alpha=.025)$. A significant simple effect of beverage was observed for the heavy memory load condition, with reduced sensitivity displayed by intoxicated relative to sober participants, $t(30)=3.45, p=.002$. No significant beverage simple effect on sensitivity was observed for the light memory load condition.

No other significant effects were observed. In particular, no significant Beverage $\times$ Block Response Frequency interaction or Beverage $\times$ Memory Load $\times$ Block Response Frequency interactions were detected.

Response bias. Means and standard errors across all conditions for the SDT $c^{\prime}$ index of response bias are displayed in the

\footnotetext{
${ }^{7}$ The indices of sensitivity and response bias we used were selected and calculated on the basis of information provided by Macmillan and Creelman (1991); $d^{\prime}$ was calculated as $z$ (hit rate $)-z$ (false alarm rate). To avoid infinite values, the conventional practice of converting hit and false alarm rate proportions of 0 and 1 to $1 /(2 N)$ and $1-1 /(2 N)$, respectively, was followed (cf. Macmillan \& Creelman, 1991, p. 10). The choice of $d^{\prime}$ to index sensitivity was made for several reasons. Specifically, it is a monotonic function of both hit and false alarm rates, it is easily interpreted as the conceptual distance between the midpoints of the match and mismatch trial distributions in standard deviation units, it is invariant when factors other than sensitivity (e.g., response bias) change, and it has ratio-scaling properties. A value of zero represents chance discrimination between match and mismatch trials, with larger values representing increased ability to discriminate between these two types of trials. The equation to calculate $c^{\prime}$ is $-0.5[z$ (hit rate $)+z$ (false alarm rate) $] /[z$ (hit rate $)-z$ (false alarm rate) $]$. It was chosen to index response bias because it is also a monotonic function of both hit and false alarm rates, its interpretation follows directly from $d^{\prime}$ as the relative threshold criterion location for response decision in standard deviation units, it is scaled to adjust for changes in discrimination across differing task conditions, and it also possesses ratio-scaling properties. A value of zero represents absence of response bias and a threshold criterion location equidistant between match and mismatch trial distributions. Increasing values (positive numbers) represent bias toward withholding response, and decreasing values (negative numbers) represent bias toward response. Macmillan and Creelman provide a comprehensive introduction to SDT and parameters

${ }^{8}$ The distributions of scores for $d^{\prime}$ within conditions were negatively skewed. However, results of analyses done after transformation to manage skewness (i.e., reflection followed by $\log$ transformation) replicated exactly the pattern of results reported in the text. In addition, supplementary analyses of the relevant simple effects using nonparametric statistics that do not make stringent assumptions about the shape of the distribution of the dependent variable further supported the meaningfulness of the $d^{\prime}$ findings. Specifically, substituting Mann-Whitney $U$ and Wilcoxon signed-rank tests for between- and within-subjects $t$ tests, respectively, also matched the reported results.
} 

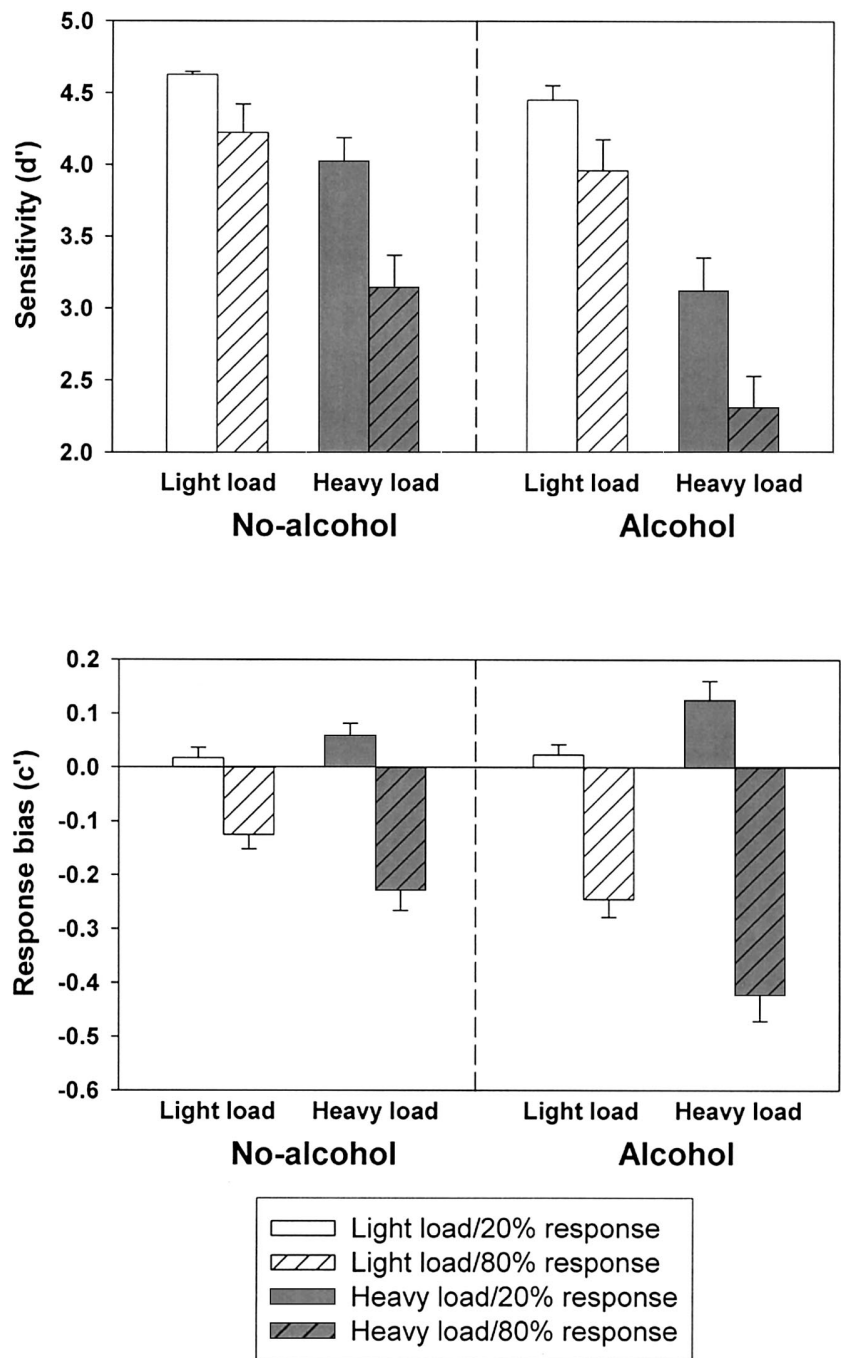

Figure 2. Mean $d^{\prime}$ index of sensitivity (top panel) and $c^{\prime}$ index of response bias (bottom panel) by beverage group, memory load (light load $=1$-back, heavy load $=2$-back), and response frequency within block.

bottom panel of Figure 2. As expected, a significant main effect of block response frequency, $F(1,30)=151.46, p<.001$, confirmed that this manipulation was successful in establishing different behavioral response sets across $20 \%$ versus $80 \%$ response frequency blocks. A $c^{\prime}$ of zero represents a response set that is balanced between tendency to respond (negative $c^{\prime}$ values) and tendency to withhold response (positive $c^{\prime}$ values). Follow-up of the main effect of block response frequency with one-sample $t$ tests against zero indicated that the mean $c^{\prime}$ in $80 \%$ response blocks $\left(c^{\prime}=-.26\right)$ was significantly less than zero, $t(31)=9.32$, $p<.001$. Mean $c^{\prime}$ in $20 \%$ response blocks $\left(c^{\prime}=.06\right)$ was significantly greater than zero $t(31)=4.07, p<.001$. However, the magnitude of the bias to respond in $80 \%$ response blocks was greater than the magnitude of the bias to withhold response in $20 \%$ response blocks, $t(31)=6.42, p<.001$.

A significant Memory Load $\times$ Block Response Frequency interaction was observed, $F(1,30)=50.94, p<.001$. Follow-up of this interaction with simple effects tests $(\alpha=.025)$ indicated that, as expected, response bias was more extreme in the heavy than light memory load condition. Specifically, a significant simple effect of memory load on response bias was observed in $20 \%$ response blocks with a greater tendency to withhold response in heavy load/20\% blocks $\left(c^{\prime}=.09\right)$ than in light load/20\% blocks $\left(c^{\prime}\right.$ $=.02), t(31)=3.19, p=.003$. Similarly, a significant simple effect of memory load on response bias was observed in $80 \%$ response blocks with a greater tendency to respond in heavy load $/ 80 \%$ blocks $\left(c^{\prime}=-.33\right)$ than in light load/80\% blocks $\left(c^{\prime}=\right.$ $-.19), t(31)=5.94, p=.003$.

With regard to beverage condition, lower-order effects of this variable were moderated by a significant beverage $\times$ memory Load $\times$ Block Response Frequency interaction, $F(1,30)=$ 5.04, $p=.032$. This three-way interaction involving beverage group indicated that the magnitude of the two-way Memory Load $\times$ Block Response Frequency interaction reported previously was stronger for participants in the alcohol group, $F(1$, $15)=36.38, p<.001$, than for participants in the no-alcohol group, $F(1,15)=15.15, p=.001$. The two-way Beverage $\times$ Memory Load interactions within each beverage group were then decomposed by conducting separate simple effects tests of memory load at each block response frequency $(\alpha=.025)$ for the two beverage groups.

Within the no-alcohol group, a significant simple effect of memory load was observed in the $80 \%$ response frequency blocks, $t(15)=2.97, p=.009$, with a greater bias to respond in the heavy versus light memory load condition. No significant effect of memory load was observed on response bias for no-alcohol participants in the $20 \%$ response blocks. Within the alcohol group, a significant simple effect of memory load was observed in the $80 \%$ response blocks, $t(15)=5.87, p<.001$, and consistent with the effect observed among no-alcohol participants, a greater bias to respond was observed in heavy load/80\% blocks than light load/80\% blocks. In addition, this effect was larger in magnitude than that observed among no-alcohol participants (difference between $c^{\prime}$ for heavy and light load conditions of .18 and .10 for alcohol vs. no-alcohol participants, respectively). Moreover, and in contrast to results for no-alcohol participants, a significant effect of memory load was also observed for alcohol participants in the $20 \%$ response blocks, $t(15)=2.76, p=.015$, with a greater response bias to withhold responding in the heavy load/20\% blocks than in the light load $/ 20 \%$ blocks.

These results indicate that under conditions of heavy memory load, the alcohol participants displayed greater response bias than no-alcohol participants. Furthermore, it is important to note that the direction of the observed response bias was dependent on the type of block. In heavy load/80\% blocks, alcohol participants displayed a greater bias toward responding. In heavy load/20\% blocks, alcohol participants displayed greater bias toward withholding response.

\section{Response Time}

To examine possible differential speed versus accuracy performance trade-offs between beverage groups, a Beverage $\times$ Memory Load $\times$ Block Response Frequency repeated-measures ANOVA 
was conducted on response time for trials with correct response. ${ }^{9}$ As expected, a main effect of memory load was observed with response time faster on low $(M=509.7, S D=109.6)$ versus high memory load conditions $(M=602.1, S D=158.2), F(1$, $30)=29.96, p<.001$. A main effect of block response frequency was also observed with response time faster on $80 \%$ response blocks $(M=535.0, S D=138.0)$ relative to $20 \%$ response blocks $(M=576.9, S D=139.5), F(1,30)=4.99, p=.033$. No main effect or interactions with beverage were observed, which indicates that the beverage effects on task accuracy reported previously cannot be attributed to differences in response speed across beverage groups. ${ }^{10}$

\section{Discussion}

The primary aim of this study was to provide an experimental evaluation of the hypothesis that perseverative behavior during intoxication results, at least in part, from specific alcohol-induced impairments in working memory and set-shifting aspects of cognitive control. This was accomplished through use of a task designed to manipulate key components of cognitive control, namely, the cognitive complexity of task demands and the prepotency of inclinations both to respond and to withhold a response. A process-oriented approach was used to help establish specific impairments in working memory and set-shifting processes as the cognitive mechanisms underlying perseverative behavior within the task. Specifically, based on Cohen's (e.g., Braver et al., 1999; MacDonald et al., 2000; Miller \& Cohen, 2001) conceptualization of cognitive control, we developed and evaluated explicit a priori predictions regarding patterns of behavioral performance and signal detection estimates of sensitivity and response bias across different beverage and task conditions. This represented a major refinement and extension of previous relevant research efforts (e.g., Fillmore et al., 1999) in that it focused explicitly on working memory and set shifting as critical elements of cognitive control. Furthermore, this study was the first to evaluate the possibility that alcohol might act to enhance not only perseveration of prepotent responding, but also perseveration of prepotent inclinations to withhold a response.

A number of potentially important results emerged from this investigation. Most notably, the overall pattern of findings was consistent with the thesis that alcohol-induced perseveration is a function of alcohol's deleterious effects on the working memory and set-shifting processes of cognitive control. Findings that support this thesis are reviewed in the following. We conclude with a brief commentary on study limitations and offer a few suggestions for future directions that research on alcohol, cognitive control, and behavioral dysregulation might take.

\section{Evidence for Alcohol-Induced Impairment in Cognitive Control}

Compelling support was obtained for the hypothesis that alcohol-induced perseveration derives from impairment of cognitive control. Pertinent evidence emerged from analyses of perseveration of prepotent inclinations both to respond and to withhold response as a function of within-block variations in response frequency and cognitive demand. These analyses revealed that the deleterious effects of alcohol on performance were greatest under conditions characterized by both (a) high cognitive complexity of task demands associated with heavy working memory load and (b) the presence of prepotent, but inappropriate, response inclinations. When one or both of these features were absent, alcohol's performance-impairing effects were markedly weaker or nonsignificant.

Specifically, in the $80 \%$ response frequency condition that encouraged commission errors, alcohol significantly exacerbated the tendency toward errors of this type, much more notably under conditions involving heavy memory load. Similarly, in the $20 \%$ response frequency condition that was conducive to omission errors, alcohol produced significantly more errors of this type, again, specifically under conditions of heavy memory load. Thus, for errors of both types, intoxication resulted in perseveration of the prevailing prepotent response set bias established by manipulation of response frequency through local block instruction (see Figure 1).

It should be noted in this context that alcohol did not produce a general impairment of task performance (i.e., increases in both commission and omission errors across all task conditions) or a selective impairment in inhibitory capacity (i.e., increases in commission errors only across all task conditions). Rather, its effects were specific to the particular types of errors occurring in the specific conditions that would be expected if such behavioral impairments occurred as a result of alcohol's deleterious impact on the set-shifting and working memory aspects of cognitive control (viz., increased commission errors in the $80 \%$ response condition, and increased omission errors in the $20 \%$ response condition, both primarily at the heavy load level).

Similarly, the specific pattern of results emerging from analyses of SDT parameters provided further evidence that alcohol's perseverative effects occurred in conjunction with specific impairment in both the working memory and set-shifting processes that are central to Cohen's (e.g., Braver et al., 1999; MacDonald et al., 2000; Miller \& Cohen, 2001) conceptualization of cognitive control. Analysis of the SDT sensitivity parameter suggested that alcohol impaired abilities necessary to discriminate between target and nontarget events, but only under heavy memory load. This effect occurred regardless of the response frequency manipulation used to establish the prepotency of a particular response option. In contrast, and as expected, alcohol's effects on the direction of the SDT response bias parameter depended upon the response frequency manipulation. Specifically, alcohol increased the bias to respond in the $80 \%$ response frequency condition, whereas it increased the bias to withhold response in the $20 \%$ response frequency condition. These effects were increased under heavy memory load conditions.

Taken together, the selective patterns of results observed in the behavioral and SDT analyses converge to provide strong support for the notion that alcohol-induced impairment in cognitive control

\footnotetext{
${ }^{9}$ No differences exist between results reported for correct response time and similar analyses conducted on response time across all trials, regardless of accuracy.

${ }^{10}$ In particular, it is important to emphasize that no main effect of beverage was observed on response time $(p=.670)$, indicating that intoxicated individuals $(M=565.8, S D=148.1)$ did not evidence an overall slowing (or speeding) of response time relative to nonintoxicated participants $(M=546.1, S D=107.7)$.
} 
processes can be a key contributor to perseverative behavior among intoxicated individuals. With this conceptualization of alcohol's effects in mind, intoxicated performance within the $n$-back task can be interpreted in terms of the response demands and memory requirements of the task and the impact of alcohol on working memory capacity.

In the $80 \%$ response condition, active responding was the automatic (default) option. Within task blocks of this type, participants responded automatically as long as no memory match occurred. Cognitive control of responding (i.e., withhold the default response) was called for only on trials involving a memory match. Under light memory (1-back) demands, the SDT sensitivity data for both beverage groups indicated that working memory processes remained largely intact. This is consistent with the notion that the "withhold to a match" instruction was maintained at a sufficient level of activation in working memory to enable participants to effectively overcome the prepotent inclination and inhibit responding on trials involving a match. Although visual inspection of Figure 1 suggests some alcohol-induced increase in commission errors under light memory demands, the difference between beverage groups was nonsignificant. In contrast, under conditions of heavy memory load (2-back), SDT sensitivity results indicated a significant decline in discriminative abilities among intoxicated individuals, suggesting an alcohol-induced decrement in working memory processes. In connection with this breakdown, it can be inferred that the withhold instruction was likely less strongly activated in intoxicated participants, so that the default (i.e., more highly activated) response remained preeminent, biasing behavior in the inappropriate direction and leading to failures to inhibit to a match.

In the $20 \%$ response condition, on the other hand, the automatic default option was to withhold responding. Within task blocks of this type, participants were biased to withhold responding automatically as long as no memory match occurred, with responses emitted only on trials involving a match. Under light memory load demands, SDT sensitivity data for both beverage groups again indicated that working memory processes remained largely intact. With these processes functioning effectively in both beverage groups, the "respond to a match instruction" apparently remained sufficiently activated in working memory to overcome the prepotent inclination to withhold and to prompt enactment of a response. In contrast, under conditions of heavy memory load, SDT sensitivity results again indicated a significant decline in discriminative abilities among intoxicated individuals, suggesting an alcoholinduced decrement in working memory processes. In connection with this breakdown, the respond instruction appeared to be less strongly activated in participants whose cognitive control capacity was impaired by alcohol, leading the default tendency to withhold responding to bias behavior the inappropriate direction, thereby causing an increase in errors of omission.

\section{Study Limitations and Directions for Future Research}

This effort to break new ground in the analysis of alcohol, cognitive control, and behavioral performance evidenced a number of limitations that could be addressed in future studies to help clarify interpretations of data from this initial study. First, interpretations such as those provided in the preceding paragraphs imply a largely neuropharmacological account of the differences between beverage groups. However, because this study did not include a placebo group, we cannot entirely rule out alcohol expectancy as a potential determinant of the observed beverage group differences. In our view, it appears unlikely that this factor would have produced the observed patterns of different error types across different task conditions, but expectancy has been known to produce complex patterns of responding in reaction time tasks (e.g., Linnoila, Stapleton, Lister, Guthrie, \& Eckardt, 1986). For example, participants' belief that alcohol tends to slow responding could lead to compensatory speeding of reaction time (RT) at the cost of decreased task accuracy, and such speed-accuracy tradeoffs have been documented in previous investigations of alcohol's impact on performance in complex choice reaction time tasks (e.g., Linnoila et al., 1986; Mitchell, 1985). However, RT data here do not support the notion of a speed-accuracy trade-off that might occur if intoxicated participants expected alcohol to slow their responses and tried to compensate. RT did not differ significantly between beverage groups in any of the task conditions. This lack of beverage group differences on RT also helps to rule out other alternative explanations for the observed beverage group differences on error rates, such as failure to understand instructions or a lack of motivation occurring within intoxicated participants, particularly under demanding task conditions (e.g., heavy memory load). Nonetheless, the fact that alcohol expectancy effects are not necessarily isodirectional with the pharmacological action of alcohol argues for inclusion of placebo controls in future studies of the impact of intoxication on cognitive-behavioral performance so that all possible contributors to observed results can be thoroughly assessed.

A second possible limitation of this study was its relatively small sample size and related constraints in statistical power that may accompany it. Although our sample size appeared to provide sufficient power to detect the predicted effects, it is possible that some weaker, but still potentially meaningful effects may have gone undetected in analyses because of this limitation. For example, review of Figure 1 suggests that, although statistically nonsignificant, alcohol may have led to a modest increase in commission errors even under light memory load. Clearly, the magnitude of this increase was not as large as that observed at the heavy load level (otherwise it too would have been significant), but recruitment of a larger sample would be helpful in determining if alcohol effects could occur even at the light memory load.

A third potential limitation of this study is that all task conditions involved a prepotent response option (i.e., either to respond or withhold a response). This precluded examination of the isolated effects of the working memory aspect of cognitive control on intoxicated behavioral performance in the absence of a prepotent response bias. Future studies using the $n$-back paradigm could use an even distribution of match and nonmatch trials at each of the two memory loads to do so. Under such conditions, one might anticipate alcohol-induced general impairment (i.e., increases in both commission and omission errors) due to alcohol-induced decreases in sensitivity, but no effect of alcohol on response bias due to the lack of a prepotent response option.

Although not necessarily a limitation, data from this study point to a fourth issue that may merit further investigation in future research. Specifically, it appeared that alcohol had a greater impact on commission errors in $80 \%$ blocks than on omission errors in $20 \%$ blocks. One possible reason for this may be that, although 
alcohol may impair the set-shifting process to increase either the bias to respond (i.e., disinhibition) or the bias to withhold a response (i.e., inhibition), depending on which inclination is stronger or more prepotent, it may have a stronger impact on the former than the latter. This would not be entirely unexpected, given the abundant observations of real-world examples of alcohol-induced disinhibition. A second possibility is that the response frequency manipulation may have had a stronger effect on elicitation of prepotency to respond in $80 \%$ blocks than on elicitation of prepotency to withhold response in $20 \%$ blocks across all subjects, not just those in the alcohol group. SDT response bias data appear to support this conclusion. Specifically, both sober and intoxicated participants displayed a significant bias toward responding in $80 \%$ blocks (although the magnitude of this effect was greater for alcohol), whereas only intoxicated participants displayed a bias to withhold response in $20 \%$ blocks. Furthermore, a review of the bottom panel of Figure 2 suggests that the magnitude of the bias to respond in $80 \%$ blocks among both intoxicated and sober participants was larger than the magnitude of the bias to withhold response among the intoxicated participants in $20 \%$ blocks. Nonetheless, future studies may be needed to address further any possibly differential effects of alcohol on disinhibition and inhibition, and potentially different brain mechanisms that may underlie them.

In addition to these four issues, understanding intoxicated behavioral dysregulation and the role of cognitive control in it could be further advanced by considering alcohol's impact on other aspects of the cognitive control construct not addressed here. For example, based on recent empirical studies (Botvinick et al., 2001; Braver et al., 1999; Carter, Botvinick, \& Cohen, 1999; Carter et al., 2000; MacDonald et al., 2000), Cohen and his colleagues have asserted that the brain system governing cognitive control likely includes two main components: (a) an evaluative component, responsible for monitoring the need for control and signaling when adjustments in control are necessary, and (b) a regulative component, responsible for activation and implementation of controlrelated processes. Our study was not designed to dissociate these two components to determine where alcohol had its sole or most profound effect. To do so, future alcohol challenge research must utilize experimental paradigms in which the relative contribution of one (or both) of these components can be directly manipulated, independent of the other component. Such paradigms have already been developed and utilized in basic research on cognitive control, and are readily available to advance our understanding of the specificity of alcohol's effect on cognitive control (e.g., Carter et al., 2000; MacDonald et al., 2000).

We believe that alcohol challenge studies designed to address directly the issues raised in this section have the potential to make important contributions to understand the cognitive mechanisms underlying alcohol-induced behavioral impairment. Ultimately, they should also inform efforts to prevent harmful behavioral effects associated with acute alcohol intoxication. This study suggests some promising links between cognitive science concepts and methods, and their applications by clinical scientists.

\section{References}

Baddeley, A. D. (1986). Working memory. Oxford, England: Oxford University Press.

Baddeley, A. D., \& Della Sala, D. (1996). Working memory and executive control. Philosophical Transactions of the Royal Society of London, Series B, 351, 1397-1404.

Botvinick, M. M., Braver, T. S., Barch, D. M., Carter, C. S., \& Cohen, J. D. (2001). Conflict monitoring and cognitive control. Psychological Review, 108, 624-652.

Braver, T. S., Barch, D. M., \& Cohen, J. D. (1999). Cognition and control in schizophrenia: A computational model of dopamine and prefrontal function. Biological Psychiatry, 46, 312-328.

Cahalan, D., Cisin, I. H., \& Crossley, H. M. (1969). American drinking practices. New Brunswick, NJ: Rutgers Center of Alcohol Studies.

Carter, C. S., Botvinick, M. M., \& Cohen, J. D. (1999). The contribution of the anterior cingulate cortex to executive processes in cognition. Reviews in the Neurosciences, 10, 49-57.

Carter, C. S., MacDonald, A. M., Botvinick, M., Ross, L. L., Stenger, V. A., Noll, D., \& Cohen, J. D. (2000). Parsing executive processes: Strategic vs. evaluative functions of the anterior cingulate cortex. Proceedings of the National Academy of Sciences, USA, 97, 1944-1948.

Chapman, L. J., \& Chapman, J. P. (1973). Disordered thought in schizophrenia. New York: Appleton-Century-Crofts.

Chapman, L. J., \& Chapman, J. P. (2001). Commentary on two articles concerning generalized and specific cognitive deficits. Journal of $A b$ normal Psychology, 110, 31-39.

Cohen, J. D., Barch, D. M., Carter, C., \& Servan-Schreiber, D. (1999). Context-processing deficits in schizophrenia: Converging evidence from three theoretically motivated cognitive tasks. Journal of Abnormal Psychology, 108, 120-133.

Conger, J. J. (1951). The effects of alcohol on conflict behavior in the albino rat. Quarterly Journal of Studies on Alcohol, 12, 1-29.

Cook, E. W., III, Atkinson, L., \& Lang, P. J. (1987). Stimulus control and data acquisition for IBM PC's and compatibles. Psychophysiology, 24, $726-727$.

Crews, T. M., \& Sher, K. J. (1992). Using adapted short MASTs for assessing parental alcoholism: Reliability and validity. Alcoholism: Clinical and Experimental Research, 16(3), 576-584.

Curtin, J. J., Patrick, C. J., Lang, A. R., Cacioppo, J. T., \& Birbaumer, N. (2001). Alcohol affects emotion through cognition. Psychological Science, 12, 527-531.

Curtin, J. J., \& Verona, E. (1996). Dose calculation for target bloodalcohol levels (BALs): A PC Windows-based application utilizing age, sex, height, weight, and drinking time. Unpublished computer program.

Fillmore, M. T., Vogel-Sprott, M., \& Gavrilescu, D. (1999). Alcohol effects on intentional behavior: Dissociating controlled and automatic influences. Experimental and Clinical Psychopharmacology, 7, 372378.

Finn, P. R., Justus, A., Mazas, C., \& Steinmetz, J. E. (1999). Working memory, executive processes and the effects of alcohol on go/no-go learning: Testing a model of behavioral regulation and impulsivity. Psychopharmacology, 146, 465-472.

Goldman-Rakic, P. S. (1992). Working memory and the mind. Scientific American, 267, 110-117.

Goldman-Rakic, P. S. (1996). The prefrontal landscape: Implications of functional architecture for understanding human mentation and the central executive. Philosophical Transactions of the Royal Society of London, Series B, 351, 1445-1453.

Green, D. M., \& Swets, J. A. (1966). Signal detection theory and psychophysics. New York: Wiley.

Haberlandt, K. (1997). Cognitive psychology (2nd ed.). Boston: Allyn \& Bacon.

Holloway, F. (1994). Low-dose alcohol effects on human behavior and performance: A review of post-1984 research (DOT/FAA/AM-94/24 Tech. Rep.). Washington, DC: Office of Aviation Medicine.

Jacoby, L. L., Jennings, J. M., \& Hay, J. F. (1996). Dissociating automatic and consciously controlled processes: Implications for diagnosis and rehabilitation of memory deficits. In D. Herman, C. McEvoy, C. Hert- 
zog, P. Hertel, \& M. K. Johnson (Eds.), Basic and applied memory research: Theory in context (Vol. 1, pp. 161-193). Hillsdale, NJ: Erlbaum.

Jacoby, L. L., Toth, J. P., \& Yonelinas, A. P. (1993). Separating conscious and unconscious influences of memory: Measuring recollection. Journal of Experimental Psychology: General, 122, 139-154.

Jellinek, E. M., \& McFarland, R. A. (1940). Analysis of psychological experiments of the effects of alcohol. Quarterly Journal of Studies on Alcohol, 1, 272-371.

Jonides, J. (1995). Working memory and thinking. In E. E. Smith \& D. N. Osherson (Eds.), Thinking: An invitation to cognitive science (Vol. 3; 2nd ed., pp. 215-265). Cambridge, MA: MIT Press.

Jonides, J., Schumacher, E. H., Smith, E. E., \& Lauber, E. J. (1997). Verbal working memory load affects regional brain activation as measured by PET. Journal of Cognitive Neuroscience, 9, 462-475.

Knight, R. A., \& Silverstein, S. M. (2001). A process-oriented approach for averting confounds resulting from general performance deficits in schizophrenia. Journal of Abnormal Psychology, 110, 15-30.

Linnoila, M., Stapleton, J., Lister, R., Guthrie, S., \& Eckardt, M. (1986). Effects of alcohol on accident risk. Pathologist, 40, 36-41.

MacDonald, A. W., Cohen, J. D., Stenger, V. A., \& Carter, C. S. (2000). Dissociating the role of the dorsolateral prefrontal and anterior cingulate cortex in cognitive control. Science, 288, 1835-1838.

Macmillan, N. A., \& Creelman, C. D. (1991). Detection theory: A user's guide. New York: Cambridge University Press

Melia, K., Corodimas, K., Ryabinin, A., Wilson, M., \& LeDoux, J. (1994). Ethanol pretreatment selectively impairs classical conditioning of contextual cues: Possible involvement of the hippocampus. Society for Neuroscience Abstracts, 24, 1007.

Miller, E. K., \& Cohen, J. D. (2001). An integrative theory of prefrontal cortex function. Annual Review of Neuroscience, 24, 167-202.
Mitchell, M. C. (1985). Alcohol-induced impairment of the central nervous system function: Behavioral skills involved in driving. Journal of Studies on Alcohol (Jul. Suppl. 10), 109-116.

Norman, D. A., \& Shallice, T. (1986). Attention to action: Willed and automatic control of behavior. In R. J. Davidson, G. E. Schwartz, \& D. Shapiro (Eds.), Consciousness and self-regulation: Vol. 4. Advances in research and theory (pp. 1-18). New York: Plenum Press.

Selzer, M. L., Vinokur, A., \& Rooijen, L. (1975). A self-administered Short Michigan Alcoholism Screening Test (SMAST). Quarterly Journal of Studies on Alcohol, 36, 117-126.

Shah, P., \& Miyake, A. (1999). Models of working memory: An introduction. In A. Miyake \& P. Shah (Eds.), Models of working memory: Mechanisms of active maintenance and executive control (pp. 1-27). New York: Cambridge University Press.

SPSS Advanced Models 10.0. (1999). Chicago, IL: SPSS

Steele, C. M., \& Josephs, R. A. (1990). Alcohol myopia: Its prized and dangerous effects. American Psychologist, 45, 921-933.

Stevens, J. P. (2002). Applied multivariate statistics for the social sciences (4th ed.). Mahwah, NJ: Erlbaum.

Strauss, M. E. (2001). Methodology for identifying specific psychological deficits: Introduction to the special section. Journal of Abnormal Psychology, 110, 4-5.

Watson, P. E. (1989). Total body water and blood alcohol levels: Updating the fundamentals. In K. Crow \& R. Batt (Eds.), Human metabolism of alcohol: Pharmacokinetics, medicolegal aspects, and general interest (Vol. 1, pp. 41-58). Boca Raton, FL: CRC Press.

Received February 26, 2002

Revision received January 31, 2003

Accepted January 31, 2003 